\title{
Arrhythmie-Mechanismus bestimmt Ablationsstrategie
}

\author{
Dank bedeutender technischer Fortschritte stellt die Katheterablation \\ heute bei den meisten ventrikulären Tachykardien eine sehr gute The- \\ rapieoption dar. Die Erfolgsraten sind unabhängig von der Grunder- \\ krankung höher als bei der medikamentösen Therapie, aber doch \\ abhängig von der Art der Grunderkrankung.
}

\begin{abstract}
B ei ventrikulären Tachykardien (VT) muss man zwei Formen unterscheiden: die „idiopathische“ bei Patienten ohne strukturelle Herzerkrankung und die VT bei struktureller Herzerkrankung, wobei es sich um eine ischämische oder nicht ischämische Herzerkrankung handeln kann. Bei „,idiopathischen“ VT kann der Ursprung im Ausflusstrakt oder in den Faszikeln liegen. „Diese Form wird immer durch einen arrhythmogenen Fokus induziert, der gezielt abladiert werden kann“, sagte Prof. Isabel Deisenhofer aus München. Bei einer strukturellen Herzerkrankung handele es sich dagegen um eine Narben-assoziierte Reentry-Tachykardie, sodass das gesamte arrhythmogene Substrat abladiert werden müsse.
\end{abstract}

\section{Idiopathische VT: fokale Ablation}

Indikationen für die Ablation einer idiopathischen VT sind:

- Symptomatische monomorphe VT oder gehäufte ventrikulären Extrasystolie, wenn $>5-10 \%$ aller Schläge ventrikuläre Extrasystolen (VES) sind,

- therapierefraktäre asymptomatische VT oder VES mit > 15\% aller Schläge, — rezidivierende polymorphe $\mathrm{VT}$, wenn eine triggernde VES existiert.

„Die Indikation bei asymptomatischen Patienten bzw. bei jenen mit ausgeprägten ventrikulären Extrasystolien ergibt sich daraus, dass sich eine Arrhythmieinduzierte Kardiomyopathie entwickeln kann, die durch das Ausschalten der VT bzw. VES verhindert wird“, so Deisenhofer. Doch eine asymptomatische VES mit $<15 \%$ aller Schläge sei keine Indikation - weder für eine Ablation noch für eine antiarrhythmische Medikation. Die Erfolgsrate der epi- oder endokardialen Katheterablation liege heute bei
Herzgesunden bei 75 bis $85 \%$.

Bei Patienten mit struktureller Herzerkrankung ist eine Ablation bei symptomatischer VT immer indiziert, auch wenn sie von einem ICD erfolgreich überstimuliert werden kann. Für Patienten, die Antiarrhythmika nicht vertragen oder diese nicht ausreichend wirken, ist die Ablation die einzige Therapieoption. „Die Katheterablation ist sinnvoller, als die antiarrhythmische Medikation zu intensivieren“, meint Deisenhofer.

Eine Indikation für die Ablation ist bei Patienten mit struktureller Herzerkrankung aber auch eine monomorphe ventrikuläre Extrasystolie, und zwar bei symptomatischen Patienten ab 5 bis $10 \%$ VES und bei asymptomatischen ab 15 bis $20 \%$ VES, um eine Kardiomyopathie zu verhindern. Bei „electrical storm“, also bei rezidivierenden VT, die medikamentös nicht beherrscht werden können, ist das Verfahren notfallmäßig indiziert.

Bei ischämischer Kardiomyopathie entstehen Zonen langsamer Leitung am Rand des Narbengewebes und in der Narbe, was den Nährboden für die Entstehung einer Reentry-Tachykardie darstellt. Dies erfordert ein Mapping von "conductive channels" und eine 3D-Darstellung der Narbe sowie ihrer arrhythmogenen „border zone“.

\section{Substrat-basierte Ablation bei struktureller Herzerkrankung}

Das Ablationsziel bei laufender ReentryVT ist die Zone der langsamen Leitung, weniger der exit point. Im Unterschied zu VT bei Herzgesunden erfordert diese bei Herzkranken eine umfassende Substratmodifikation. „Nicht nur die aktuell aufgetretene VT, sondern das gesamte potenziell arrhythmogene Narbengewebe muss abladiert werden", so Deisen- hofer. In einer Studie ließ sich die Erfolgsrate verdoppeln, wenn Substratbasiert abladiert wurde, statt nur die klinisch manifeste VT anzugehen. Die Erfolgsaussichten der Ablation sind auch bei Herzkranken überzeugend: $65 \%$ der Patienten hatten gar keine oder deutlich weniger VT-Ereignisse. Die Ablation ist der medikamentösen Therapie somit überlegen, sowohl in der Senkung der VT-Last als auch der kompletten Arrhythmie-Elimination. Sie ist auch durchführbar bei geringer Ejektionsfraktion (EF) und wenn keine weitere Revaskularisation mehr möglich ist. Auch war die primär-prophylaktische Ablation in einer randomisierten Studie vor der ICD-Implantation einer medikamentösen Prophylaxe überlegen. „VT bei Patienten mit stabiler KHK lassen sich nicht ,weg-stenten', sondern sollten abladiert werden“, so Deisenhofer.

\section{Schlechtere Ergebnisse bei nicht ischämischer Kardiomyopathie}

Bei nicht ischämischer Kardiomyopathie lassen sich fibrotische Areale nicht so eindeutig lokalisieren wie bei der ischämischen. Dies erklärt die schlechteren Erfolgsraten. Ein ereignisfreies Überleben fand sich bei 57 bzw. $43 \%$ der Patienten mit ischämischer Kardiomyopathie nach einem Jahr bzw. drei Jahren, dagegen nur bei 41 bzw. $23 \%$ der Patienten mit nicht ischämischer Kardiomyopathie. „Daher sollte man die Entscheidung für die Ablation hier individuell treffen“, empfiehlt Deisenhofer.

sti

Quelle: Cardiovascular Summit, 24.9.2016, Köln

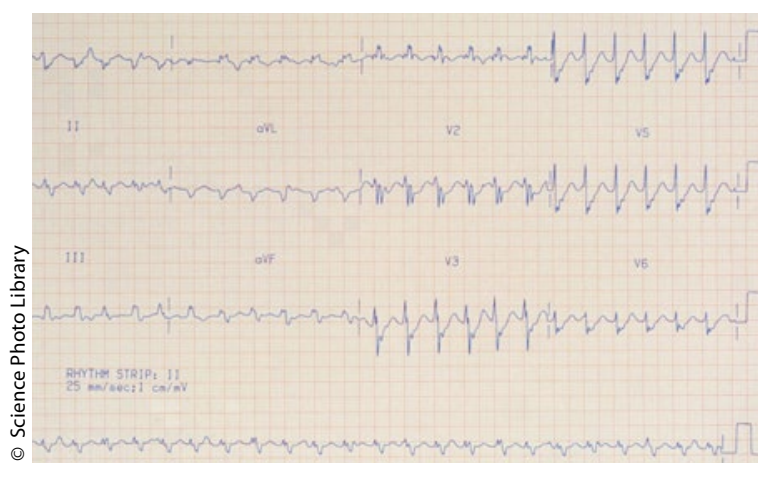

Das EKG deutet auf eine Kardiomyopathie-bedingte ventrikuläre Tachykardie hin. Dann sollte das gesamte arrhythmogene Narbengewebe abladiert werden. 